STEPHANI BYZANTII ETHNICA 


\title{
CORPUS FONTIUM \\ HISTORIAE BYZANTINAE
}

CONSILIO SOCIETATIS INTERNATIONALIS STUDIIS BYZANTINIS PROVEHENDIS DESTINATAE EDITUM

\author{
VOLUMEN XLIII/4
}

SERIES BEROLINENSIS

EDIDIT

ATHANASIOS KAMBYLIS

DE GRUYTER

BEROLINI ET BOSTONIAE

MMXVI 


\title{
STEPHANI BYZANTII ETHNICA VOLUMEN IV: П-Y
}

RECENSUERUNT GERMANICE VERTERUNT ADNOTATIONIBUS INDICIBUSQUE INSTRUXERUNT

\author{
MARGARETHE BILLERBECK \\ ET \\ ARLETTE NEUMANN-HARTMANN
}

DE GRUYTER

BEROLINI ET BOSTONIAE

MMXVI 
ISBN 978-3-11-021967-8

e-ISBN (PDF) 978-3-11-021968-5

e-ISBN (EPUB) 978-3-11-038474-1

\section{Library of Congress Cataloging-in-Publication Data}

A CIP catalog record for this book has been applied for at the Library of Congress.

Bibliografische Information Der Deutschen Nationalbibliothek.

Die Deutsche Nationalbibliothek verzeichnet diese Publikation in der Deutschen Nationalbibliografie; detaillierte bibliografische Daten sind im Internet über http://dnb.dnb.de abrufbar.

\section{(C) 2016 Walter de Gruyter GmbH, Berlin/Boston}

Einbandgestaltung: Christopher Schneider, Laufen

Satz: Dörlemann Satz GmbH \& Co. KG, Lemförde

Druck und buchbinderische Verarbeitung: Hubert \& Co. GmbH \& Co. KG, Göttingen $\infty$ Gedruckt auf säurefreiem Papier,

Printed in Germany

www.degruyter.com 